\title{
Torsion of the Vascular Pedicle of A Wandering Spleen: An Unusual Cause of Intestinal Strangulation
}

\author{
Randimbinirina Zakarimanana Lucas, Ratsimarisolo Nathan, Rajaonarivony Maheriandrianina \\ Fanambinana Voahary, Ahmad Ahmad, and Ravalisoa Marie Lydia Agnès
}

\section{ABSTRACT}

Ectopic spleen is a rare splenic malformation. Apart from torsion of the vascular pedicle, a common complication, an ectopic spleen could be responsible of an acute intestinal obstruction. We report a case of an 8-yearold girl who presented an acute abdomen, following of the transverse colon strangulation by the torsion of the vascular pedicle of a wandering spleen. The patients benefited a surgical opening into the abdomen who has been confirmed the diagnosis and made a detorsion of the vascular pedicle of spleen with splenopexy.

The interest of this case report lies on the rarity of a wandering spleen and on the unusual situation of transverse colon strangulation by the vascular pedicle of spleen.

Keywords: CT-scan, intestinal obstruction, torsion, vascular pedicle, wandering spleen.

\author{
Published Online: April 30, 2021 \\ ISSN: $2736-5476$ \\ DOI : $10.24018 /$ ejclinicmed.2021.2.2.51 \\ Randimbinirina Zakarimanana \\ Lucas* \\ Department of Surgery, Faculty of \\ Medicine of Antananarivo, Madagascar \\ (e-mail: lucaszakarimanana@ gmail.com) \\ Ratsimarisolo Nathan \\ Department of Radiology, Faculty of \\ Medicine of Antananarivo, Antananarivo \\ Rajaonarivony Maheriandrianina \\ Fanambinana Voahary \\ Department of Surgery, Faculty of \\ Medicine of Antananarivo, Madagascar \\ Ahmad Ahmad \\ Department of Radiology, Faculty of \\ Medicine of Antananarivo, University of \\ Antananarivo, Madagascar \\ Ravalisoa Marie Lydia Agnès \\ Department of Surgery, Faculty of \\ Medicine of Antananarivo, University of \\ Antananarivo, Madagascar \\ *Corresponding Author
}

\section{INTRODUCTION}

Wandering spleen is defined as mobile spleen that is attached only by an elongated vascular pedicle, allowing it to migrate to any part of the abdomen or pelvis. It's a rare clinical entity with an estimated incidence of $0.2 \%$ [1]. The torsion of a wandering spleen is a surgical emergency. Delayed of surgical treatment could be exposed the spleen on infarction and about $10 \%$ of the spleen's infarction ended with a splenectomy [2]. However, an acute intestinal obstruction following torsion of wandering spleen is an uncommon situation [3]. Few cases have been reported in the literature. We report a case of an 8-year-old girl who presented an acute intestinal obstruction following of transverse colon strangulation by the vascular pedicle of a wandering spleen.

\section{CASE PRESENTATION}

An 8-year-old girl was referred for paroxysmal abdominal pain since 5 days ago, associated with vomiting and stop of stool and gas. She was seen by a regular doctor who prescribed analgesics and antispasmodics but no improvement was noted. Pain is becoming more and more frequent and violent. At the entrance in Paediatric Emergency, the child was very agitated, complaining of diffuse abdominal pain. The abdominal palpation showed a generalized defense. Biology showed hyperleukocytosis (GB:27,370/mm3) with neutrophilic polynucleosis (PNN: 90\%). The C-Reactive Protein was elevated to $162 \mathrm{mg} / \mathrm{l}$. Abdominal-pelvic ultrasound showed a few quantity of intraperitoneal fluid effusion associated with a large bowel distension and splenomegaly. The abdominal-pelvic CT without injection of iodized contrast material showed an image of dilatation of bowel. The spleen was ectopic, located on the left flank compressing a part of the descending colon. There was a vacuity in the splenic lodge in left hypochondriac region (Figure 1). 


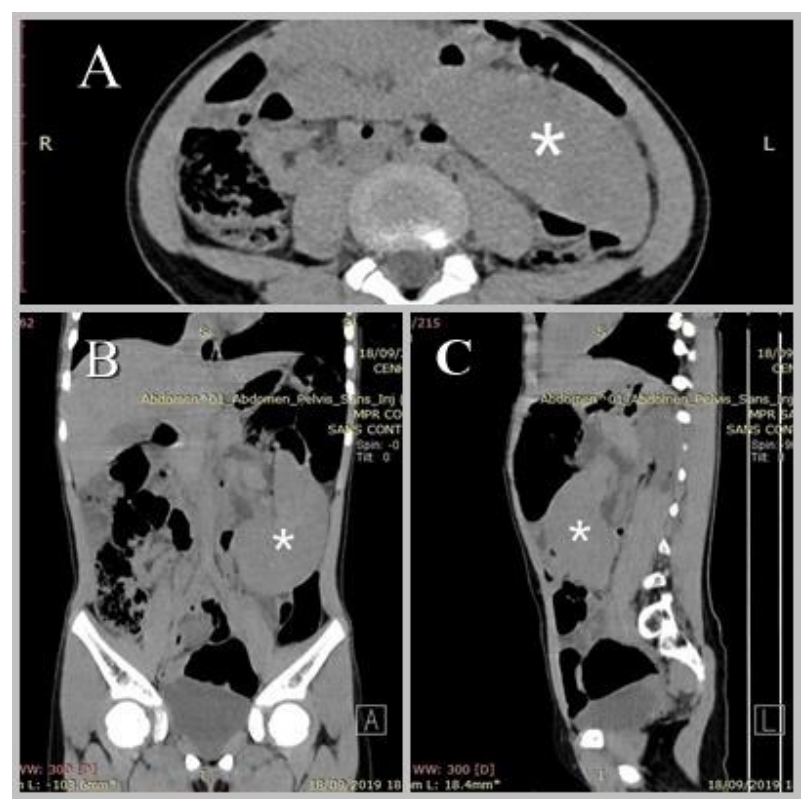

Fig. 1. Multiplanar reconstruction (A: axial view, B: coronal view, C: sagittal view) of an abdominal and pelvic CT without injection of iodized contrast material showing an ectopic spleen (white asterisk)

An emergency laparotomy has been performed for this acute abdomen. During exploration, the ileum and the right segment of colon were dilated. The spleen was increased in volume, ischemic, located in left flank. The transverse colon has been strangulated by the torsion of vascular pedicle of spleen (Figure 2). The vascular pedicle of spleen was rotated for 4 rounds (Figure 3).

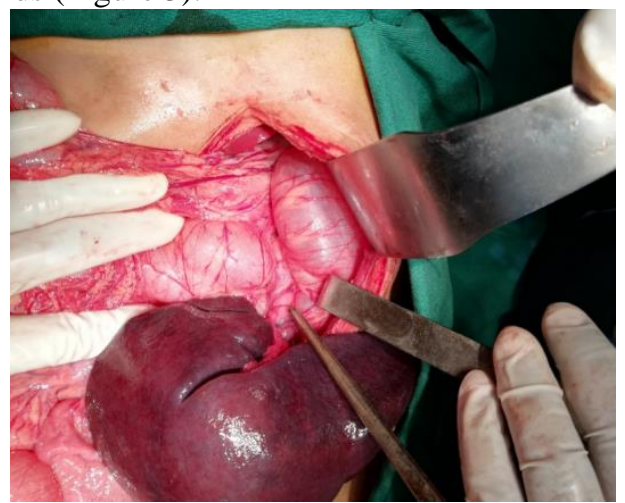

Fig. 2. Strangulation of the transverse colon by the twisted splenic pedicle

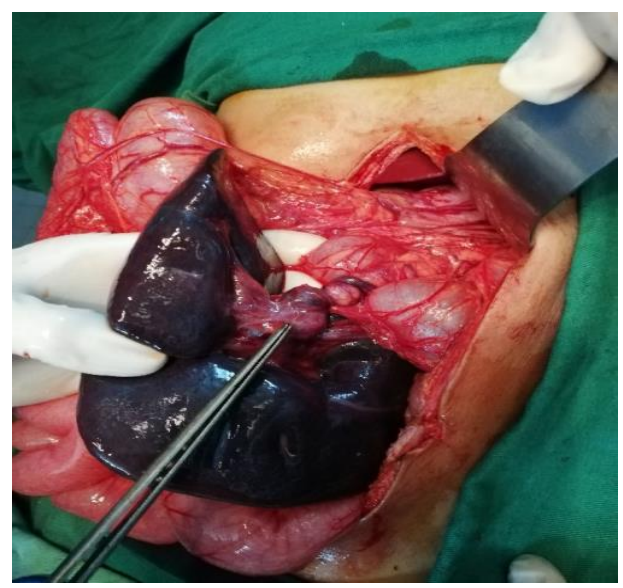

Fig. 3. Chronic torsion of the vascular pedicle of spleen.

Manual detorsion of vascular pedicle of spleen was performed, following by splenopexy. The postoperative evolution was simple. There is no early postoperative complication. One year later, the abdominal-pelvic CT control showed an image of hypotrophy of spleen located in the left hypochondrium, with $4.97 \mathrm{~cm}$ of axial dimension (Figure 4).

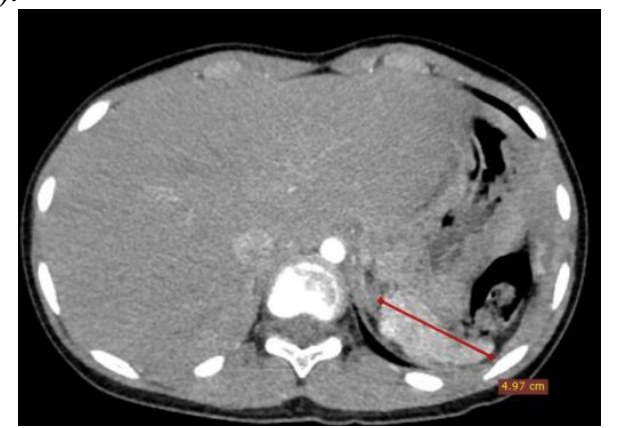

Fig. 4. Hypotrophic spleen located in left hypocondrium in axial view of an abdominal and pelvic CT

\section{DISCUSSION}

The wandering of spleen results of a hyperlaxity of the suspensory ligaments of the spleen. This hyperlaxity of splenic ligaments suspensory is an uncommon situation, related to congenital or acquired abnormalities, responsible for a hypermobility and migration of the spleen outside of his normal anatomical compartment. The wandering spleen is a rare clinical entity [1]. The torsion of the vascular pedicle of spleen is the most complication of the wandering spleen. This torsion of the vascular pedicle of spleen constitute 0.2 to $0.3 \%$ of the reason for splenectomy [4,5]. It usually occurs in children and young adults. A female predominance has been reported in the literature with an average age of 8 years [1]. In our case, it is an 8-years-old girl.

The clinical diagnosis of the wandering spleen is difficult. The ectopic spleen can remain asymptomatic for a long time. It could be move around in all the different quadrants of the abdomen [3]. The circumstances of diagnosis are either fortuitous during abdominal palpation or imaging requested for other reasons, or during acute abdominal pain secondary to the torsion of the vascular pedicle [4,6]. In our case, the symptom was the intense abdominal pain associated with signs of peritoneal irritation. This problem of intense abdominal pain explained the indication of an exploratory laparotomy. However, Germain has been reported a case of a wandering spleen revealed by a subocclusive intestinal syndrome [3]. In our case, the compression of the descending colon by the wandering spleen and strangulation of the transverse colon by the vascular pedicle of spleen could explained the acute intestinal obstruction. The palpation of the abdomen quadrants could be showed an abdominal mass. However, the presence of bowel dilatation could make difficult the palpation of the splenic mass. The presence of an abdominal guarding is very suggestive of a torsion of the wandering spleen in the most case report $[4,6]$.

Medical imaging allows the preoperative diagnosis of a wandering spleen. The diagnosis can be confirmed by abdominal ultrasound, abdominal pelvic CT scan or abdominal magnetic resonance imaging (MRI). In addition, Chen has been reported the advantage of an abdominal ultrasound for the diagnosis of a subclinical wandering spleen [7]. Abdominal ultrasound could confirm the diagnosis by showing the vacuity of the splenic lodge. Abdominal 
ultrasound are using to measure the spleen's dimension, to evaluate the echogenicity of the splenic parenchyma. In Doppler ultrasound, the decrease or missing of blood flow in splenic artery or spenic vein are suggestive of torsion of vascular pedicle $[7,8]$. In our case, the diagnosis of splenic torsion by abdominal ultrasound was difficult due to abdominal air. In otherwise, CT-scan could help the clinician for the diagnosis of wandering spleen if the clinical signs is not sufficient. The torsion of vascular pedicle of spleen could observed on CT-scan [9]. According to Khan's study, CTscan is the best method of choice for diagnosis when splenic torsion is suspected [10].

According the anatomopathological point of view, the wandering spleen could be located in the different quadrants of the abdomen: hypogastric [6,10], epigastric [11], periumbilical [5], left iliac fossa [12], right flank [3]. In our case, the spleen is located in the left flank.

When the diagnosis of torsion of splenic vessel has been made, surgical exploration must be done urgently. Delayed treatment exposes the spleen to the risk of splenic infarction. Exploratory laparotomy is necessary to confirm the diagnosis and make a better therapeutic decision. So, if there is not a necrosis of the spleen, detorsion of the vascular pedicle could be performed associated with a splenopexy to keep the spleen in his lodge. According to Alqadi's study, laparoscopic splenopexy is feasible in $90 \%$ of cases in children [2]. However, splenectomy must be done if there is a necrosis of spleen secondary of delayed of surgical management [13].

\section{CONCLUSION}

The torsion of the vascular pedicle of a wandering spleen is a rare cause of an acute surgical abdomen. His diagnosis could be made with the presence of acute abdominal pain associated with a mobile abdominal mass. In addition, it's exceptionally possible that the bowel segment was strangulated by the vascular pedicle of the wandering spleen. Ultrasound or abdominal-pelvic CT- scan could help the clinician to confirm the diagnosis. The optimal treatment is the splenopexy after detorsion of the vascular pedicle of spleen. Splenectomy is indicated if there is an infarction of the spleen.

\section{CONFLICTS OF INTEREST}

All authors have declared that they have no conflict of interest in the submitted work.

\section{REFERENCES}

[1] Heba Taher, Mostafa Abdellatif, Mahmoud Tarek, Gamal El Tagy. Torsion of wandering spleen in an infant associated with hamartomatous vascular malformation. J Pediatr Surg Case Rep 2019;40:13-16.

[2] Alqadi GO, Saxena AK. Is laparoscopic approach for wandering spleen in children an option?. J Min Access Surg 2019;15:93-7.

[3] Germain M. Une torsion de rate baladeuse révélée par un syndrome subocclusif intestinal. Ann Afr Med 2018;11(4):e3051

[4] Kapan M, Gumus M, Onder A, Gumus H. A wandering spleen presenting as an acute abdomen: Case report. J Emerg Med 2012;43(5):e303-e305.

[5] Nwashilli JN, Ezeokenwa MO, Ukwuoma JK. Wandering spleen causing recurrent abdominal pain. Niger J Surg Sci 2015;25:37-40.
[6] Khalid E, Fatimazahra B, Rachid L, Abdelaziz F, Mohamed L, Nadia $\mathrm{B}$ et al.. Acute abdomen in a torsion of an ectopic spleen: report of a case. Pan Afr Med J 2012;11:62-62.

[7] Chen JW, Yeh DM., Peng SH, Chen GS, Tseng YH, Lin CW et al. Sonographic diagnosis of a subclinical wandering spleen: role of the decubitus position. J Ultrasound Med 2012;31(3):483-487.

[8] Rasheed B, Ahmed F, Iqbal Z, Babar S, Ahmed N. Wandering spleen with tortion leading to infarction. Europ J Radiol Extra 2011;77(1):57.

[9] Puglisi F, Potenza A, Melchionda M, Capuano P, De Fazio M, Lobascio P, Martines G, Lograno G. Wandering spleen as a cause of acute abdomen: a case report. Chir Ital 2005;57(3):373-375.

[10] Khan D B, Khandwala K, Abbasi S, et al. Torsion of Wandering Spleen with Infarction. Cureus 2018;10(8):e3177.

[11] Dème H, Akpo LG, Fall S, Badji N, Ka I, Guèye ML et al. Torsion chronique d'une rate baladeuse chez un adolescent: à propos d'un cas. Pan Afr Med J 2016;24(1).

[12] Ashok Kumar Puranik. Wandering spleen: a surgical enigma. Gastroenterol Rep 2017;5(3):241-243.

[13] Singla V, Galwa RP, Khandewal N, Poornachandra KS, Dutta U, Kochhar R. Wandering spleen presenting as bleeding gastric varices. Am J Emerg Med 2008;26(5):637.

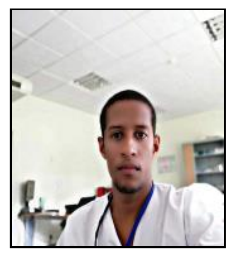

Randimbinirina Zakarimanana Lucas, is cardiovascular surgeon in Soavinandriana Hospital Center in Antananarivo, Madagascar. 